Burnham: None declared, Julia G. Harris: None declared, Judyann C. Olson: None declared, Mileka Gilbert: None declared, Michelle Batthish Speakers bureau: Novartis, Abbvie, Michael Shishov: None declared, Dustin Fleck: None declared, Esi Morgan: None declared DOI: 10.1136/annrheumdis-2019-eular.4395

\section{FRI0551 IDENTIFYING RESEARCH PRIORITIES FOR JUVENILE IDIOPATHIC ARTHRITIS TOGETHER WITH PATIENTS, CARERS AND CLINICIANS THROUGH THE JAMES LIND ALLIANCE METHOD: PRELIMINARY RESULTS}

Anouk Verwoerd ${ }^{1}$, Wineke Armbrust ${ }^{2,3}$, Katherine Cowan ${ }^{4}$, Wendy Olsder ${ }^{5}$, Jeannette Cappon ${ }^{6,7}$, Casper Schoemaker ${ }^{1,8}$. ${ }^{1}$ Wilhelmina Children's Hospital (WKZ), Department of Paediatric Rheumatology, Utrecht, Netherlands; ${ }^{2}$ Beatrix Children's Hospital, Department of Paediatric Rheumatology, Groningen, Netherlands; ${ }^{3}$ Dutch Association for Paediatric Rheumatology, Amsterdam, Netherlands; ${ }^{4}$ James Lind Alliance, Southampton, United Kingdom; ${ }^{5}$ Youth-R-Well. com, member of EULAR Young PARE, Eindhoven, Netherlands; ${ }^{6}$ Reade, Centre for Rehabilitation and Rheumatology, Amsterdam, Netherlands; ${ }^{7}$ Dutch Health Professionals in Paediatric Rheumatology, Amsterdam, Netherlands; ${ }^{8} \mathrm{JIA}$ patient and parent organisation, member of ENCA, Amsterdam, Netherlands

Background: Research on Juvenile Idiopathic Arthritis (JIA) should have the primary goal to ultimately improve the lives of the affected patients, and help health professionals provide the best care for them. Therefore, these end users of research evidence - patients, carers (parents/caregivers) and clinicians - should be included in the process of identifying research priorities. Importantly, patients and carers can use their unique experiential knowledge from living with the disease to give vital input to researchers in designing a study. Combining this input with the goal of research(ers) to make a true impact on patients' lives, will result in research that is more effective and meaningful, and thereby increases research value [1]. To this end, we initiated the project of establishing a research agenda for JIA jointly by patients, carers and clinicians [2].

Objectives: The objective of this project is to bring patients, carers and clinicians together to identify research priorities and create a research agenda for JIA, such that future research into JIA is guided to the issues that matter most.

Methods: The method for research priority setting developed by the James Lind Alliance (JLA) is used [3]. This method consists of several steps with the end goal of creating a top 10 list of research priorities agreed on by all parties. First, a steering group with equal representation of patients, parents and clinicians was assembled. We are now in the process of collecting research questions through an online survey. Focus groups are being held to ensure the inclusion of younger patients. Questions will be clustered and checked against the evidence. Next, the process of interim priority setting will follow, in which a shortlist of up to 30 questions will be assembled. During a final workshop in which all parties are equally represented, the top 10 research priorities will be established. This workshop is scheduled for the end of 2019. A process evaluation is conducted to monitor ethical aspects in the decision-making process.

Results: The first step of collecting research questions is performed until the end of March 2019. We will be able to present preliminary results on the questions gathered from the online survey and focus groups by June 2019. Furthermore, we will discuss the effects of our efforts to be inclusive and representative of every party involved.

Conclusion: Through this project, patients, carers and clinicians are brought together to define the most important unanswered questions for JIA. Engaging patients and stakeholders in this crucial phase is the next step in performing more meaningful and effective research.

\section{REFERENCES:}

[1] Chalmers I, Bracken MB, Djulbegovic BD, et al. How to increase value and reduce waste when research priorities are set. Lancet 2014; 383;156165.

[2] Schoemaker CG, Armbrust W, Swart JF, et al. Dutch juvenile idiopathic arthritis patients, carers and clinicians create a research agenda together following the James Lind Alliance method: a study protocol. Pediatr Rheumatol Online J 2018;16(1):57.

[3] James Lind Alliance. Guidebook. 2018; Available from: www.jla.nihr.ac.uk/ jla-guidebook. Accessed 4 January 2018.

Acknowledgement: We thank all the members on the steering group of the JIA Priority Setting Partnership.

Disclosure of Interests: None declared

DOI: 10.1136/annrheumdis-2019-eular.993
FRI0552 PERFORMANCE OF NEWLY PROPOSED PERIODIC FEVER, APHTHOUS STOMATITIS, PHARYNGITIS AND CERVICAL ADENITIS (PFAPA) SYNDROME CRITERIA IN REGIONS ENDEMIC FOR FAMILLIAL MEDITERRANEAN FEVER (FMF)

Amra Adrovic ${ }^{1}$, Mehmet Yildiz², Ipek Ulkersoy ${ }^{2}$, Neslihan Gucuyener ${ }^{2}$, Melisa Kanber ${ }^{2}$, Oya Koker², Sezgin Sahin', Kenan Barut², Ozgur Kasapcopur? ${ }^{1}$ Istanbul University-Cerrahpasa, Cerrahpasa Medical School, Pediatric Rheumatology, Istanbul, Turkey; ${ }^{2}$ Istanbul University-Cerrahpasa, Cerrahpasa Medical School, Pediatric Rheumatology, Istanbul, Turkey

Background: The periodic fever, aphthous stomatitis, pharyngitis, and cervical adenitis (PFAPA) syndrome is an autoinflammatory condition characterized by regularly recurrent episodes of high fever lasting 3 to 6 accompanied by aphthosis, cervical adenitis, and pharyngitis, in the absence of upper respiratory tract infections. A relevant number of patients with monogenic periodic fevers also meet the diagnostic criteria for PFAPA syndrome. Especially in regions endemic for familial Mediterranean fever (FMF), it would be important to avoid useless genetic testing and to be able to identify PFAPA patients by using clinical classification criteria. Despite the high specificity, widely used Marshall's criteria have been shown to have low specificity. An international consensus among experts has been established recently, in order to define a set of classification criteria for PFAPA syndrome with a better performance in term of sensitivity and specificity.

Objectives: We aimed to evaluate the performance of recently proposed PFAPA criteria, in order to assess their utility in regions endemic for FMF.

Methods: Patients diagnosed with PFAPA syndrome, FMF and juvenile idiopathic arthritis (JIA) were consecutively included in the study. PFAPA diagnosis has been established by Marshall's criteria. Patients with FMF and JIA were diagnosed according to Turkish pediatric FMF criteria and ILAR criteria, respectively. Two investigators blindly evaluated all of patients for the newly proposed PFAPA criteria.

Results: A total of 321 PFAPA, 118 FMF and 45 JIA patients with mean age of $7.23 \pm 2.9,14.7 \pm 3.09,13.5 \pm 4.6$ years, respectively, were included in the study. A $45 \%(146 / 323)$ of PFAPA, $50 \%(59 / 118)$ of FMF and $58 \%$ $(23 / 45)$ of JIA patients were female. We found quite high sensitivity $(90 \%)$ of newly proposed PFAPA criteria: 289 out of $321(90 \%)$ patients followed up as PFAPA syndrome fulfilled newly proposed PFAPA criteria, as well. When applied to patients diagnosed with FMF and JIA, 46 out of $118(39 \%)$ FMF and 10 out of $45(22 \%)$ JIA patients also fulfilled newly proposed PFAPA criteria. Specificity of recently proposed PFAPA criteria was found to be $61 \%$ and $77 \%$, among FMF and JIA patients, respectively. Positive predictive value was $86 \%$ and $97 \%$, negative predictive value was $69 \%$ and $50 \%$ for FMF and JIA patients, respectively. Conclusion: Recently proposed PFAPA criteria have satisfactory high sensitivity. Specificity of recently proposed criteria is still under expectation in regions endemic for FMF. Multicentric studies with higher patients' number in different regions are needed in order to provide more relevant data on performance of newly proposed PFAPA criteria.

\section{REFERENCES:}

[1] Marshall GS, Edwards KM, Butler J, Lawton AR. Syndrome of periodic fever, pharyngitis, and aphthous stomatitis. J Pediatr. 1987;110(1):43- 46

[2] Adrovic A, Sahin S, Barut K, Kasapcopur O. Familial Mediterranean fever and periodic fever, aphthous stomatitis, pharyngitis, and adenitis (PFAPA) syndrome: shared features and main differences. Rheumatol Int. 2019;39 (1):29-36.

[3] Vanoni F, Federici S, Antón J, et al. An international delphi survey for the definition of the variables for the development of new classification criteria for periodic fever aphtous stomatitis pharingitis cervical adenitis (PFAPA) Pediatr Rheumatol Online J. 2018;16(1):27

[4] Vanoni F, Caorsi R, Aeby S, et al. Towards a new set of classification criteria for PFAPA syndrome. Pediatr Rheumatol Online J. 2018;16(1):60

[5] Pehlivan E, Adrovic A, Sahin S, et al. PFAPA Syndrome in a Population with Endemic Familial Mediterranean Fever. J Pediatr. 2018;192:253-255.

Disclosure of Interests: None declared

DOI: 10.1136/annrheumdis-2019-eular.2262 